\title{
Spotting ignition of fuel beds by firebrands
}

\author{
C. Lautenberger \& A. C. Fernandez-Pello \\ Department of Mechanical Engineering, University of California, \\ Berkeley, CA, USA
}

\begin{abstract}
Wind can carry fire-lofted embers or molten/burning metal particles generated by powerline interactions long distances, where they may land on and ignite fuel beds remote from the source. This process, known as spotting, is a common mechanism of wildland and wildland urban interface fire propagation. The physical processes leading to spot fire initiation after an ember or heated particle has landed are not yet quantitatively understood. To provide insight into spot fire initiation, this paper presents a comprehensive 2D numerical model for the potential ignition of a porous fuel bed by an ember or hot metal particle. The model consists of a computational fluid dynamics (CFDs) representation of the gas-phase coupled to a heat transfer and pyrolysis model that simulates condensed-phase phenomena. The coupled model is used to simulate ignition of a powdered cellulose porous fuel bed by glowing pine embers in a laboratory experiment. The model provides qualitative information regarding the mechanisms that lead to ignition, smolder, or flame propagation on a porous fuel bed that agree qualitatively with experimental observations. This work provides the foundation for a more complete study of the problem where the effects of different factors (moisture content, humidity, temperature, porosity, particle size/heat content, etc.) are quantified.
\end{abstract}

Keywords: spotting, embers, ignition.

\section{Introduction}

Firebrand spotting is a primary mechanism for the spread of both wildland and wildland-urban-interface (WUI) fires. Spotting can lead to rapid fire spread because firebrands generated by burning vegetation are lofted by the fire plume and transported downwind to ignite secondary fires or structures far from the fire front. In addition to propagation by firebrand spotting, many wildland fires are 
initiated by heated or burning metallic particles generated from different sources such as powerline interactions or conductor clashing in high winds, overheated catalytic converters, and hot work/welding. The three primary steps in formation of spot fires are: 1) Firebrand/metal particle generation; 2) Firebrand lofting and ember/particle transport; 3) Ignition (or non-ignition) of fuels after a firebrand/particle lands.

Of these, the aspect of the spot fire formation process that is least understood is what happens after a firebrand or heated particle lands on a target fuel bed, i.e. whether or not flaming ignition (or smoldering ignition followed by transition to flaming) occurs. This highly complex process depends on several factors including: size and state of the brand (smoldering/glowing, flaming), characteristics of the fuel bed on which it lands (temperature, density, porosity, moisture content), and environmental conditions (temperature, humidity, wind velocity). Three types of ignition mechanisms that may occur are: 1) Smoldering ignition; 2) Piloted gas-phase ignition induced by the brand or particle itself; and 3) Prolonged smolder followed by spontaneous transition to flaming. Ignition of fuels by fire brands and heated surfaces has been studied primarily experimentally, in particular by workers at NIST [1-4] and Jones [5-7] applied "hot spot" theory to investigate the problem analytically. Zvyagils'kaya and Subbotin [8] and Grishin et al. [9] applied numerical models that considered a porous condensed-phase that represented natural vegetation. However, there have been few modeling studies that include a porous condensed-phase model (to simulate the target fuel bed) coupled to a gas-phase code (to simulate the exterior "ambient"). This coupled approach is required to faithfully simulate the three ignition mechanisms described above, and considerable progress is still needed before models reach this point and can be considered predictive. The objective of this work is to develop a model to simulate the smoldering ignition of powdered cellulose fuel beds by glowing pine embers. The source code, executable files, and sample input files are freely available through an opensource project known as Gpyro [10].

\section{Model description}

\subsection{Physical configuration}

The physical configuration modeled here is the ember-initiated smolder of a powdered cellulose fuel bed with air flowing over its surface. In addition to simulating this physical problem with a computer model, a few qualitative laboratory experiments were conducted, making it possible to qualitatively compare the behavior predicted by the model to that seen experimentally. To introduce the physical configuration simulated here, the experiments are described briefly below.

The bench-scale test apparatus consists of a small-scale wind tunnel $38 \mathrm{~cm}$ in length, $13.5 \mathrm{~cm}$ in width, and $8 \mathrm{~cm}$ in height. Powdered cellulose is placed in an aluminum sample holder that is $12 \mathrm{~cm}$ in length with a $4 \mathrm{~cm}$ by $4 \mathrm{~cm}$ cross section. The sample is conditioned in an oven at $110^{\circ} \mathrm{C}$ for approximately 1 hour 
to remove most moisture near the surface before. To reduce heat losses, the sample holder is lined with fiberglass insulation (3 $\mathrm{mm}$ thickness) and then embedded flush in the bottom wall of the wind tunnel.

Compressed air flows through a converging duct into the test section at a prescribed velocity. An experiment begins by dropping a firebrand (ember) onto the powdered cellulose. Embers are generated by immersing pine cylinders of different sizes in a premixed propane flame for $40 \mathrm{~s}$. Glowing embers are dropped on the target fuel surface after flaming combustion has ceased.

The qualitative information from the experiment is used to help formulate the model and verify its predictive capabilities.

In this work, a two dimensional "slice" down the centerline of the experimental apparatus is modeled. Use of a 2D (instead of 3D) computational domain significantly reduces the required CPU time. A 2D computational domain is commensurate with the qualitative nature of simulations at this early stage of model development. The computational domain used in the modeling is shown in Figure 1. To limit CPU and storage requirements, a subsection of the wind tunnel $25.6 \mathrm{~cm}$ in length is modeled (total length of the wind tunnel is 38 $\mathrm{cm})$.

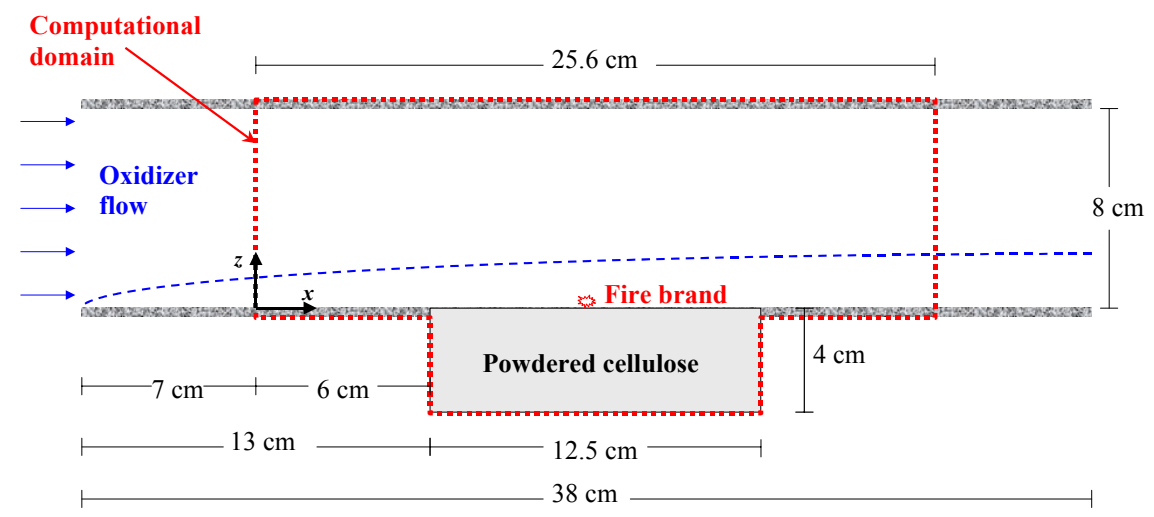

Figure 1: Computational domain.

The model description that follows is split into three parts: 1) Condensedphase, which applies inside the powdered cellulose, 2) Gas-phase, which applies in the exterior ambient, and 3) Boundary/initial conditions.

\subsection{Condensed-phase (porous fuel bed)}

The condensed-phase computational model formulation includes the twodimensional conservation equations for a combustible porous material undergoing thermal and oxidative reactions. The equations are solved numerically, with details given in [11]. 


\subsubsection{Governing equations}

Assumptions inherent in the condensed-phase model formulation are given in [11]. The resultant two-dimensional conservation equations (and auxiliary relations such as Darcy's law and the ideal gas law) that apply inside the powdered cellulose are (see $[10,11]$ for details):

$$
\begin{gathered}
\frac{\partial \bar{\rho}}{\partial t}=-\dot{\omega}_{f g}^{\prime \prime \prime} \\
\frac{\partial\left(\bar{\rho} Y_{i}\right)}{\partial t}=\dot{\omega}_{f i}^{\prime \prime \prime}-\dot{\omega}_{d i}^{\prime \prime \prime} \\
\frac{\partial\left(\rho_{g} \bar{\psi}\right)}{\partial t}+\frac{\partial \dot{m}_{x}^{\prime \prime}}{\partial x}+\frac{\partial \dot{m}_{z}^{\prime \prime}}{\partial z}=\dot{\omega}_{f g}^{\prime \prime \prime} \\
\frac{\partial\left(\rho_{g} \bar{\psi} Y_{j}\right)}{\partial t}+\frac{\partial\left(\dot{m}_{x}^{\prime \prime} Y_{j}\right)}{\partial x}+\frac{\partial\left(\dot{m}_{z}^{\prime \prime} Y_{j}\right)}{\partial z}=-\frac{\partial \dot{j}_{j, x}^{\prime \prime}}{\partial x}-\frac{\partial \dot{j}_{j, z}^{\prime \prime}}{\partial z}+\dot{\omega}_{f j}^{\prime \prime \prime}-\dot{\omega}_{d j}^{\prime \prime \prime} \\
\frac{\partial(\bar{\rho} \bar{h})}{\partial t}+\frac{\partial\left(\dot{m}_{x}^{\prime \prime} h_{g}\right)}{\partial x}+\frac{\partial\left(\dot{m}_{z}^{\prime \prime} h_{g}\right)}{\partial z}=-\frac{\partial \dot{q}_{x}^{\prime \prime}}{\partial x}-\frac{\partial \dot{q}_{z}^{\prime \prime}}{\partial z}+\dot{Q}_{s}^{\prime \prime \prime}+\sum_{i=1}^{M}\left(\dot{\omega}_{f i}^{\prime \prime \prime}-\dot{\omega}_{d i}^{\prime \prime \prime}\right) h_{i} \\
\dot{q}_{x}^{\prime \prime}=-\bar{k} \frac{\partial T}{\partial x} \quad \dot{q}_{z}^{\prime \prime}=-\bar{k} \frac{\partial T}{\partial z} \\
\dot{m}_{g}^{\prime \prime}=T \\
\bar{K} \frac{\partial P}{v} \frac{\dot{m}_{j}}{\partial x} \quad \dot{m}_{z}^{\prime \prime}=-\frac{\bar{K}}{v} \frac{\partial P}{\partial z} \\
\rho_{g}=\frac{P M}{R T_{g}}
\end{gathered}
$$

The pressure is determined by substituting Eq. (8) and Eq. (7) into Eq. (3). and solving the resultant pressure evolution equation. In the equations above, a subscript $i$ refers to the condensed-phase and a subscript $j$ refers to the gas-phase. An overbar denotes a weighted or averaged quantity, i.e. $\bar{k}=\sum X_{i} k_{i}$. See Lautenberger [11] for details.

\subsubsection{Source terms}

The governing equations presented in the previous section contain several source terms attributed to chemical reactions $\left(\dot{\omega}_{f i}^{\prime \prime \prime}, \dot{\omega}_{d i}^{\prime \prime \prime}, \dot{\omega}_{f j}^{\prime \prime \prime}, \dot{\omega}_{d i}^{\prime \prime \prime}, \dot{\omega}_{f g}^{\prime \prime \prime}\right.$, and $\left.\dot{Q}_{s}^{\prime \prime \prime}\right)$ that must be quantified. These source terms are presented below in generalized form.

Heterogeneous reaction stoichiometry is written in general form as:

$$
1 \operatorname{kg} A_{k}+\sum_{j=1}^{N} v_{j, k}^{\prime} \operatorname{kg} \text { gas } j \rightarrow v_{B, k} \operatorname{kg} B_{k}+\sum_{j=1}^{N} v_{j, k}^{\prime \prime} \operatorname{kg} \text { gas } j
$$




$$
v_{B_{k}}=\frac{\rho_{B_{k}}}{\rho_{A_{k}}}
$$

Each reaction $k$ converts a condensed-phase species having index $A_{k}$ to a condensed-phase species having index $B_{k}$. Gases may be consumed or produced in the process. The destruction rate of condensed-phase species $A_{k}$ by reaction $k$ is calculated as either thermal or oxidative pyrolysis:

$$
\begin{gathered}
\dot{\omega}_{d A_{k}}^{\prime \prime \prime}=\left(\frac{\bar{\rho} Y_{A_{k}}}{\left.\bar{\rho} Y_{A_{k}}\right)_{\Sigma}}\right)^{n_{k}}\left(\bar{\rho} Y_{A_{k}}\right)_{\Sigma} Z_{k} \exp \left(-\frac{E_{k}}{R T}\right)\left(\text { for } n_{\mathrm{O}_{2}, k}=0\right) \\
\dot{\omega}_{d A_{k}}^{\prime \prime \prime}=\left(\frac{\bar{\rho} Y_{A_{k}}}{\left(\bar{\rho} Y_{A_{k}}\right)_{\Sigma}}\right)^{n_{k}}\left(\bar{\rho} Y_{A_{k}}\right)_{\Sigma}\left[\left(1+Y_{\mathrm{O}_{2}}\right)^{n_{\mathrm{O}_{2}, k}} Z_{k} \exp \left(-\frac{E_{k}}{R T}\right)\left(\text { for } n_{\mathrm{O}_{2}, k} \neq 0\right)\right.
\end{gathered}
$$

The formation rate of condensed-phase species $B_{k}$ by reaction $k$ is related to condensed-phase bulk density ratios as:

$$
\dot{\omega}_{f B_{k}}^{\prime \prime \prime}=v_{B, k} \dot{\omega}_{d A_{k}}^{\prime \prime \prime}=\frac{\rho_{B_{k}}}{\rho_{A_{k}}} \dot{\omega}_{d A_{k}}^{\prime \prime \prime}
$$

The formation rate of all gases (conversion rate of condensed-phase mass to gasphase mass) by reaction $k$ is:

$$
\dot{\omega}_{f g_{k}}^{\prime \prime \prime}=\left(1-v_{B, k}\right) \dot{\omega}_{d A_{k}}^{\prime \prime \prime}=\left(1-\frac{\rho_{B_{k}}}{\rho_{A_{k}}}\right) \dot{\omega}_{d A_{k}}^{\prime \prime \prime}
$$

The formation and destruction rates of gaseous species $j$ from condensed-phase reaction $k$ are calculated as:

$$
\begin{aligned}
& \dot{\omega}_{f j, k}^{\prime \prime \prime}=v_{j, k}^{\prime \prime} \dot{\omega}_{d A_{k}}^{\prime \prime \prime}=\dot{\omega}_{f g, k}^{\prime \prime \prime} \max \left(y_{s, j, k}, 0\right) \\
& \dot{\omega}_{d j, k}^{\prime \prime \prime}=v_{j, k}^{\prime} \dot{\omega}_{d A_{k}}^{\prime \prime \prime}=-\dot{\omega}_{f g, k}^{\prime \prime \prime} \min \left(y_{s, j, k}, 0\right)
\end{aligned}
$$

where $y_{s, j, k}$ is the $N$ by $K$ species yield matrix, see Lautenberger [11] for details. Associated with each reaction $k$ is a heat of reaction:

$$
\dot{Q}_{s, k}^{\prime \prime \prime}=-\dot{\omega}_{f g_{k}}^{\prime \prime \prime} \Delta H_{v o l, k}
$$

The total source terms appearing in the conservation equations are obtained by summing over all reactions. 


\subsubsection{Numerical solution methodology}

The governing equations described above yield a system of coupled algebraic equations that are solved numerically. Due to the nonlinearity introduced by the source terms and temperature-dependent thermophysical properties, a fullyimplicit formulation is adopted for solution of all equations. The gas-phase species, gas-phase momentum, and condensed-phase energy conservation equations are solved using a computationally efficient tridiagonal matrix algorithm (TDMA). The two-dimensionality of the governing equations is handled using a line-by-line TDMA. The condensed-phase mass and condensedphase species conservation equations are solved with a customized fully implicit solver that uses relaxation to prevent divergence. Convective terms are fully upwinded. Additional details are given in [11]. The condensed-phase uses a nominal $1 \mathrm{~mm}$ by $1 \mathrm{~mm}$ grid spacing. The timestep is set by the gas-phase code to satisfy the CFL condition required for stability.

\subsubsection{Reaction mechanism and material properties}

The reaction mechanism used here is based on a mechanism developed previously to simulate the oxidative pyrolysis of white pine [11]. Since here the powdered cellulose samples are dried before conducting an experiment, the moisture evaporation step is excluded, and the mechanism consists of three steps:

$$
\begin{gathered}
\text { cellulose } \rightarrow v_{\text {char }} \text { char }+v_{\text {tp }} \text { thermal pyrolysate } \\
\text { cellulose }+v_{\mathrm{O}_{2} \text { cell }} \mathrm{O}_{2} \rightarrow v_{\text {char }} \text { char }+v_{\text {op }} \text { oxidative pyrolysate } \\
\text { char }+v_{\mathrm{O}_{2} \text { char }} \mathrm{O}_{2} \rightarrow v_{\text {ash }} \text { ash }+v_{\text {cop }} \text { char oxidation products }
\end{gathered}
$$

The $v$ coefficients in Eq. (15) are related to bulk density ratios and the species yield matrix discussed earlier (see Eq. (9) and Eq. (13)).

\subsection{Gas-phase (exterior ambient)}

The pyrolysis model described in Section 2.2 is coupled to Fire Dynamics Simulator (FDS) Version 5.1.3 [12], where it is applied as a boundary condition. The gas-phase equations solved by FDS and the solution methodology are described in detail in the FDS Technical Reference [12]. When applying FDS in this paper, the following simplifications and approximations are made:

- 2D elliptic flow

- Gas-phase dynamic viscosity is the molecular value (rather than the effective value calculated from the Smagorinsky model)

- Single-step irreversible Arrhenius combustion reaction

The FDS gas-phase routines are modified only minimally in this work (to permit coupling to the condensed-phase and facilitate specification of a volumetric heat source representing a glowing ember) so the reader is referred to the FDS Technical Reference [12] for complete details of the gas-phase model. 


\subsection{Boundary and initial conditions}

The boundary and initial conditions on the gas-phase (handled by FDS) and the powdered cellulose (handled by the pyrolysis model discussed in Section 2.2) are described below. Due to its importance in the simulations, the boundary condition applicable to the ember is also discussed.

\subsubsection{Boundary conditions}

For the gas-phase calculation (exterior ambient), the upper wall of the duct is modeled as an FDS 'INERT' boundary condition (impermeable wall with temperature maintained at the ambient value of $20{ }^{\circ} \mathrm{C}$ ). Air is introduced from the left by a prescribed velocity boundary condition $(0.5 \mathrm{~m} / \mathrm{s})$ with specified gasphase mass fractions ( 0.23 for oxygen, 0.77 for nitrogen, and 0 for the remaining gases), and gases leave to computational on the right via an FDS 'OPEN' boundary condition. Referring to Figure 1, the boundary condition at the bottom wall is modeled as steel for $0 \mathrm{~cm}<x<6 \mathrm{~cm}$ and $18.5 \mathrm{~cm}<x<25.6 \mathrm{~cm}$. For these solid surfaces, the gas solid coupling is handled directly by FDS. The boundary condition at the bottom wall is powdered cellulose for $6 \mathrm{~cm}<x<18.5$ $\mathrm{cm}$ and the coupling between FDS and the powdered cellulose is described in greater detail below.

For the powdered cellulose, the three bounding surfaces that do not abut the gas-phase are modeled as impermeable and perfectly insulated. The powdered cellulose abuts the gas-phase exterior ambient at $x=0$. At this interface, there is full coupling between the powdered cellulose (simulated using the pyrolysis model described earlier) and the gas-phase (simulated using FDS). That is, the temperature of the powdered cellulose is calculated by the pyrolysis model and passed to FDS. Similarly, the convection or diffusion of gas-phase species into our out of the powdered cellulose is calculated by the pyrolysis model and passed to FDS as a mass flux.

\subsubsection{Initial conditions}

The gas-phase is initially quiescent (zero velocity) with a temperature of $20{ }^{\circ} \mathrm{C}$ and a background pressure of $101.3 \mathrm{kPa}$. The initial gas-phase mass fractions are 0.23 for oxygen, 0.77 for nitrogen, and zero for the remaining species. The powdered cellulose is also initially at a temperature of $20{ }^{\circ} \mathrm{C}$. The initial condensed-phase mass fractions are 1.0 for cellulose, 0.0 for char, and 0.0 for ash.

\subsubsection{Ember model}

The ember is treated as a volumetric heat source. The order of magnitude of the volumetric heat source is estimated as:

$$
\dot{Q}^{\prime \prime \prime} \approx \frac{\left(\rho_{v}-\rho_{a}\right) \Delta H_{c}}{t_{b}} \approx \frac{400 \mathrm{~kg} / \mathrm{m}^{3} \times 10 \mathrm{MJ} / \mathrm{kg}}{900 \mathrm{~s}} \approx 4 \mathrm{MW} / \mathrm{m}^{3}
$$

where it is assumed that the brand leaves an ash with a density $\left(\rho_{a}\right)$ that is negligible in comparison to that of the virgin brand $\left(\rho_{v} \approx 400 \mathrm{~kg} / \mathrm{m}^{3}\right)$, the brand is 
completely consumed in 15 minutes $\left(t_{b}=900 \mathrm{~s}\right)$, and the average heat of combustion is $10 \mathrm{MJ} / \mathrm{kg}$ (lower than typical values for wood due to an assumed incompleteness of combustion). This is a crude estimate of the heat release rate per unit volume of a glowing ember, so $\dot{Q}^{\prime \prime \prime}$ is treated as a parameter.

\section{Results}

Two different ignition source strengths (representing the ember) are investigated here: $4 \mathrm{MW} / \mathrm{m}^{3}$ and $6 \mathrm{MW} / \mathrm{m}^{3}$. The air velocity and temperature are respectively $20^{\circ} \mathrm{C}$ and $0.5 \mathrm{~m} / \mathrm{s}$. The first case shows that the temperatures are low enough that minimal smoldering occurs, with a thin char layer forming only near the cellulose surface where it abuts the heat source representing that ember. In the second simulation, the ignition source strength is increased by $50 \%$, causing considerable smolder to occur. This leads to gas-phase ignition after $\sim 55 \mathrm{~s}$. Figure 2 shows the calculated gas-phase temperatures at $60 \mathrm{~s}$ (approximately $5 \mathrm{~s}$ after gas-phase ignition) and at $90 \mathrm{~s}$ (approximately $35 \mathrm{~s}$ after gas-phase ignition). The flame has started to spread both upstream (against the oncoming flow) and downstream (in the same direction as the oncoming flow). This is qualitatively consistent with experiments conducted with flaming brands that show flame propagation both upstream and downstream.

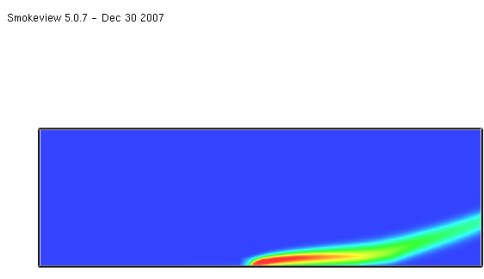

(a)

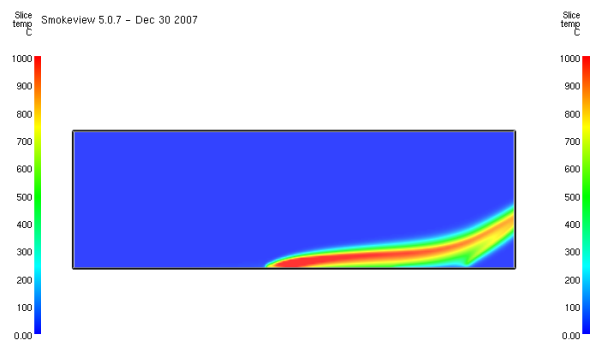

(b)

Figure 2: $\quad$ Gas-phase temperatures for $6 \mathrm{MW} / \mathrm{m}^{3}$ ember. (a) $60 \mathrm{~s}$; (b) $90 \mathrm{~s}$.

The condensed-phase temperature profile is shown in Figure 3 at the same times shown in Figure 2. Five seconds after ignition, the calculated temperature contour is similar to that for the $4 \mathrm{MW} / \mathrm{m}^{3}$ ember at $60 \mathrm{~s}$ but the heated area is larger due to the $50 \%$ greater heat release rate of the ignition source. By $35 \mathrm{~s}$ after ignition) the size of the heated region has increased considerably due convective and radiative heating from the gas-phase flame.

This model calculates the concentrations of various gas-phase species inside the decomposing porous solid (cellulose in this case). This is critical for predicting the transition from smolder to flaming as well as accounting for differences in burning behavior in inert and oxidative environments. 


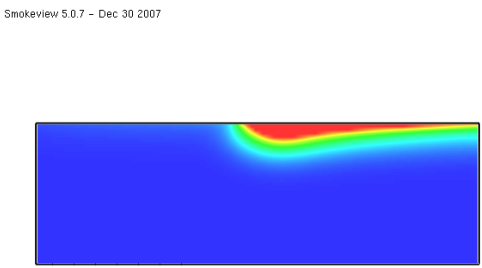

(a)

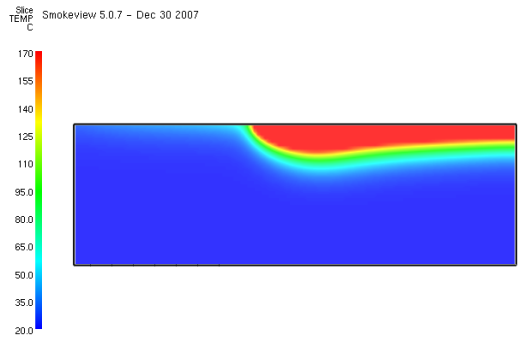

(b)

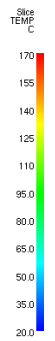

Figure 3: Condensed-phase temperatures for $6 \mathrm{MW} / \mathrm{m}^{3}$ ember. (a) $60 \mathrm{~s}$; (b) $90 \mathrm{~s}$.

\section{Concluding remarks}

The model and resultant computer simulations presented here appear to be capable of discerning between conditions that will or will not lead to initiation of a spot fire after landing of an ember. Additional work is required to characterize practical materials and to better understand the boundary condition between the ember or heated particle and the target fuel bed. Particularly challenging is determining the material properties and reaction kinetics of various fuels that must be supplied as input to the model.

\section{Acknowledgements}

This work was funded by NSF under Award 0730556, "Tackling CFD Modeling of Flame Spread on Practical Solid Combustibles". The authors would like to thank Sonia Fereres for setting up and running the experiments.

\section{References}

[1] Manzello, S.L., Cleary, T.G., Shields, J.R., Maranghides, A., Mell, W., and Yang, J.C., "Experimental Investigation of Firebrands: Generation and Ignition of Fuel Beds," to appear in Fire Safety Journal (2008).

[2] Manzello, S.L., Cleary, T.G., Shields, J.R., and Yang, J.C., "On the ignition of fuel beds by firebrands," Fire and Materials 30: 77-87 (2006).

[3] Manzello, S.L., Cleary, T.G., Shields, J.R., and Yang, J.C., "Ignition of mulch and grasses by firebrands in wildland-urban interface fires," International Journal of Wildland Fire 15: 427-431 (2006).

[4] Pitts, W., "Ignition of Cellulosic Fuels by Heated and Radiative Surfaces," NIST Technical Note 1481, March 2007.

[5] Jones, J.C., "Predictive Calculations of the Effect of an Accidental Heat Source on a Bed of Forest Litter," Journal of Fire Sciences 11: 80-86 (1993). 
[6] Jones, J.C., "Further Calculations Concerning the Accidental Supply of Heat to a Bed of Forest Material," Journal of Fire Sciences 12: 502-505 (1994).

[7] Jones, J.C., "Improved Calculations Concerning the Ignition of Forest Litter by Hot Particle Ingress," Journal of Fire Sciences 13: 350-356 (1995).

[8] Zvyagils'kaya, A.I. and Subbotin, A.N., "Influence of Moisture Content and Heat and Mass Exchange with the Surrounding Medium on the Critical Conditions of Initiation of Surface Fire," Combustion, Explosions, and Shock Waves 32: 558-564 (1996).

[9] Grishin, A.M., Dolgov, A.A., Zima, V.P., Kryuchkov, D.A., Reino, V.V., Subbottan, A.N., and Tsvyk, R. Sh., "Ignition of a Layer of Combustible Forest Materials," Combustion, Explosions, and Shock Waves 34: 613-620 (1998).

[10] http://code.google.com/p/gpyro

[11] Lautenberger, C.W., "A Generalized Pyrolysis Model for Combustible Solids," PhD Dissertation, Department of Mechanical Engineering, University of California, Berkeley, 2007.

[12] http://repositories.cdlib.org/cpl/fs/LautenbergerPhD/

[13] McGrattan, K., Hostikka, S., Floyd, J., Baum, H., and Rehm, R., "Fire Dynamics Simulator (Version 5) Technical Reference Guide," NIST Special Publication 1018-5, 2007. 